\title{
Urban Distribution Vehicle Routing Optimization and Empirical Analysis under the Influence of Carbon Trading Policy
}

\author{
Jing Wang* \\ Management School \\ Wuhan University of Science and Technology \\ China \\ *Corresponding author
}

\author{
Kang Pu, Zhuzhi Shen \\ Management School \\ Wuhan University of Science and Technology \\ China
}

\begin{abstract}
With the continuous improvement of the economic level, the carbon emission produced by logistics and distribution has become more and more neglecting. Therefore, this article is aimed at the actual distribution case of Wuhan Hongxu Information Technology Co. Ltd, combined with the impact of carbon trading policies. On the basis of the traditional vehicle routing problem model, an urban distribution vehicle routing optimization model with the goal of minimizing the total cost of the sum of time cost, fuel consumption cost, departure cost, and carbon transaction cost is established. The gene tic algorithm was used to simulate the actual delivery case of Wuhan Hongxu Information Technology Co. Ltd. The minimum cost of the distribution task was determined, the number of delivery vehicles and the route of the delivery vehicle were obtained, and the influence of the fluctuation of carbon trading parameters on the distribution cost was analyzed
\end{abstract}

Keywords-urban distribution; carbon trading; VRP; genetic algorithm

\section{INTRODUCTION}

At present, with the improvement of the carbon trading policy, manufacturing enterprises begin to consider using the existing carbon trading mechanism to reduce the carbon emission of logistics distribution, optimize the cost of enterprises, and obtain economic benefits or expend certain carbon excess cost to maintain the efficiency of distribution.

Wuhan hongxu information technology co., LTD. is a service provider of mobile communication equipment production, sales and implementation, and annual production capacity exceeding 3 billion Yuan. This enterprise implements the joint production mode, and its production and distribution mode is single distribution center and multiple distribution points. There are many joint processing enterprises near the production base, whose production and distribution are mainly self-distribution, and the distribution cost is directly undertaken by the enterprise. When logistics departments make the distribution path decisions, the logistics department regards the production base as the distribution center and the joint processing enterprise as the distribution point when making the distribution path decision.

The targets for production and distribution are as follows:
(1) The least use of the distribution vehicles;

(2) The shortest distribution time;

(3) The least consumption of distribution fuel;

(4) Distribute the least carbon emissions.

This study changes above four goals into costs to minimize the total distributed cost of enterprises and optimize the urban logistics distribution path.

\section{MODELING}

\section{A. Problem description}

Production and distribution in manufacturing industry is a path optimization problem for discussing single distribution center and multiple distribution points: The distribution service of multiple distribution points is undertaken by a distribution center, several vehicles are dispatched from the distribution center, go through all the distribution points once, and finally return to the distribution center. Assumptions are as follows:

a) All distribution vehicles are departed from the distribution center, and immediately return to the distribution center after the delivery task is completed.

b) The vehicles are the same type and the loading capacity is known.

c) The demand of each distribution point is determined, and the loading capacity of the distribution vehicle meets the customers' demand.

d) All distribution points are served and can only be served once by one car.

e) All distribution paths are road transportation with its road condition remains the same during the distribution task.

f) Vehicle carbon emission depends on vehicle fuel consumption, which is related to vehicle travel distance, load capacity and speed. 
g) The objective function considers vehicle fuel consumption cost, time cost, starting cost and carbon trading cost.

\section{B. Symbols and decision variables}

We define $G=(V, A)$ is distribution network; $V=\{0,1,2 \cdots n\}$ is a collection of all nodes, where the node 0 represents the distribution center, others represent the distribution points ; $A=\{(i, j) i \neq j, i, j \in V\}$ is an arc set ; $d_{i j}$ is the path distance between nodes $\mathrm{i}$ and $\mathrm{j}$; Each distribution point $i$ has a demand $q_{i}$, a service time $t_{i}$. The collection of vehicles is $K=\{1,2,3 \cdots m\}$; $\mathrm{Q}$ is the maximum load of the vehicle, and the standard speed of the vehicle is v; Each arc $(i, j)$ has a road condition damping factor $\mu_{i j} \geq 1$, the smaller $\mu_{i j}$ is ,the higher the traffic capacity of the road section , It means no traffic on this road section when $\mu_{i j}=+\infty$; the time cost per unit vehicle is denoted $\delta$; the cost per unit fuel consumption is denoted $\lambda$; the cost per unit vehicle is denoted $\rho ; \eta$ is the carbon price, and $P$ means carbon quotas ; where $x_{i j k}$ is a $0-1$ decision variable such that it is 1 if vehicle $k$ passes the $\operatorname{arc}(i, j)$,otherwise, $x_{i j k}=0 ; y_{i k}$ also is a $0-1$ decision variable such that it is 1 if the demand for the distribution point $i$ is completed by the vehicle $k$ ( 0 otherwise).

\section{Calculation of fuel consumption and carbon trading cost}

Literature [3][8] was introduced to calculate fuel consumption and carbon emissions. We define $\beta_{0}$ is the fuel consumption per unit mileage when the vehicle is unloaded ;The effect of extra load on the fuel consumption of the vehicle's driving unit mileage is denoted $\beta_{1}$; The effect of vehicle speed on fuel consumption per unit mileage is denoted $\beta_{2}$; The load of the vehicle traffic $\operatorname{arc}$ is $W_{i j}$; Carbon emission coefficient of fuel is $e$.

Therefore, fuel consumption of vehicle passing $\operatorname{arc}(i, j)$ is shown in equation (1)

$$
O_{i j}=\left[\beta_{0}+\beta_{1} W_{i j}+\beta_{2}\left(\frac{\mu_{i j}}{v}\right)^{2}\right] \cdot d_{i j}
$$

The carbon emission of the vehicle pass arc is distributed as formula (2)

$$
E_{i j}=O_{i j} \cdot e=\left[\beta_{0}+\beta_{1} W_{i j}+\beta_{3}\left(\frac{\mu_{i j}}{v}\right)^{2}\right] \cdot d_{i j} \cdot e
$$

The carbon transaction cost is related to the three factors of carbon quota, carbon price and carbon emission. Therefore, the carbon transaction cost function is shown in equation (3).

$$
C_{T}=\eta\left(e \sum_{i=0}^{n} \sum_{j=0}^{n} \sum_{k=1}^{m} x_{i j k}\left[\beta_{0}+\beta_{1} W_{i j}+\beta_{2}\left(\frac{\mu_{i j}}{v}\right)^{2}\right] d_{i j}-P\right)
$$

\section{Model building}

This paper aims at minimizing the total cost of distribution, considering the time cost (driving time and service time) and use-cost of the distribution, as well as the fuel consumption cost and carbon transaction cost calculated from the above as the total cost, the urban distribution route optimization model is constructed as follows:

$$
\begin{aligned}
& \min C=\lambda O+\delta\left(\sum_{i=0}^{n} \sum_{j=0}^{n} \sum_{k=1}^{m} \frac{\mu_{i j} x_{i j k} d_{i j}}{v}+\sum_{i=1}^{n} t_{i}\right)+\rho \sum_{i=1}^{n} \sum_{k=1}^{m} x_{0 i k}+\eta(O e-P) \\
& O=\sum_{i=0}^{n} \sum_{j=0}^{n} \sum_{k=1}^{m} x_{i j k}\left[\beta_{0}+\beta_{1} W_{i j}+\beta_{2}\left(\frac{\mu_{i j}}{v}\right)^{2}\right] d_{i j} \\
& \left(\sum_{i=0}^{n} \sum_{k=1}^{m} x_{i j k}=1 \quad \forall j \in V^{\prime}\right. \\
& \sum_{j=0}^{n} \sum_{k=1}^{m} x_{i j k}=1 \quad \forall i \in V^{\prime} \\
& \sum_{i=0}^{n} x_{i r k}-\sum_{j=0}^{n} x_{r j k}=0 \quad \forall r \in V, k \in K \\
& \sum_{i=1}^{n} q_{i} \sum_{j=0}^{n} x_{i j k} \leq Q \sum_{i=1}^{n} \sum_{k=1}^{m} x_{0 i k} \quad \forall k \in K \\
& \sum_{i=1}^{n} q_{i} y_{i k} \leq Q \quad \forall k \in K \\
& \sum_{i=1}^{n} x_{i 0 k}=\sum_{j=1}^{n} x_{0 j k}=1 \quad \forall k \in K \\
& x_{i j k}=[0,1] \quad y_{i k}=[0,1] \quad(i, j) \in A
\end{aligned}
$$

The objective function (4) contains four costs, the first is the time cost, the second is the fuel consumption cost, the third cost is the departure cost, and the fourth cost is the carbon transaction cost.

Constraint (5) requires that each distribution point can be served only once by one vehicle.

Constraint (6) requires that each vehicle must leave the distribution point after reaching a distribution point.

Constraint (7) requires that the total demand of all distribution points is less than or equal to the total load of all delivery vehicles.

Constraint (8) requires that the demand for all distributions on each path is not greater than the load on the vehicle.

Constraint (9) requires that the constraint of distribution center and all vehicles can only leave and return to the distribution center once

Constraint (10) is 0-1 Constraint and there are only two possibilities for a vehicle to pass or not pass a certain path and a distribution point.

\section{ALGORITHM DESIGN}

The Genetic Algorithm (GA) is a computational model that simulates the natural evolution of Darwin's biological evolution theory and the biological evolution process of genetic 
mechanism. It is a method to search for optimal solutions by simulating natural evolutionary processes. Genetic algorithm has been successfully applied to the study of classical vehicle routing problems, so this paper uses genetic algorithm as the algorithm for solving the model. The specific process is shown in Fig. 1.

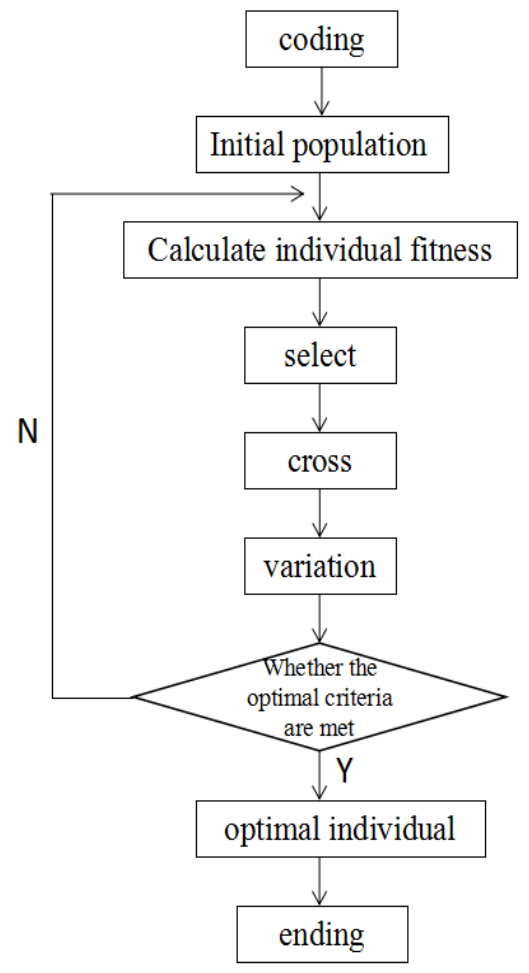

Fig. 1. Flow chart of genetic algorithm

(1) Coding. We use natural number arrangement coding. Randomly generate a code of length $(n-1)+n$, where $n$ is the natural number encoding 1-10 of the distribution point and (n-1) is the separator " 0 ”. For example code strings " $0-1-0-0-4-0-0-3-$ 5-6-0-7-9-0-0-10-8-2-0" states that 5 distribution routes for 5 cars, namely $0-1-0,0-4-0,0-3-5-6-0,0-7-9-0,0-10-8-2-$ 0 ,Where the header and footer separator 0 represents the distribution center.

(2) Initial population generation. In order to make sure that the distribution center delivers more than or equal to the demand of a customer, each individual should be created by a random method, which is a solution to the problem of the study.

(3) Individual fitness measures. The system evaluates the optimal performance of each chromosome through the adaptation function $f(x)$, so as to determine the probability of chromosome individuals to obtain crossover and mutation. This paper uses the reciprocal representation of the objective function, ie $f(x)=\frac{1}{C}$

(4) Selection operation. The selection operation is to select excellent individuals from the current group, so that they have the opportunity to be the descendants of the next generation. This paper chooses the roulette selection method.
The higher the individual's fitness, the greater the chance of being selected.

(5) Cross-operation, that is, the code of the individual is crossed with a certain probability, and the partial code of the two parent individuals is replaced and recombined to generate a new individual. This paper uses the sequential crossover operation.

(6) Mutation operation refers to changing the individual code chain according to a certain probability and changing some codes on the individual code chain.

(7) Loop iteration. After the cross mutation operation, the chromosome population has changed, thereby generating a problem of further calculating the individual fitness, so the algorithm will transfer from this step to the iterative operation until the algorithm termination condition is reached, that is, the total cost is the smallest.

\section{EMPIRICAL ANALYSIS}

\section{A. Empirical description}

This paper empirically analyzes the production and distribution cases of Wuhan Hongxu Information Technology Co., Ltd. The data in this paper is derived from the urban distribution demand arising from one of its joint production activities.

\section{1) Distribution data}

TABLE I. DISTRIBUTION SCALE(KG)

\begin{tabular}{|c|c|c|c|c|c|c|}
\hline Distribution Quantity & $q_{1}$ & $q_{2}$ & $q_{3}$ & $q_{4}$ & $q_{5}$ & \\
\hline Weight(Kg) & 1200 & 600 & 800 & 400 & 300 & \\
\hline Distribution Quantity & $q_{6}$ & $q_{7}$ & $q_{8}$ & $q_{9}$ & $q_{10}$ & $q_{S}$ \\
\hline Weight(Kg) & 500 & 1200 & 1100 & 800 & 800 & 7600 \\
\hline
\end{tabular}

2) Distance data

In this paper, the length of the vehicle's driving path on the road is estimated according to the actual map ratio of $1: 100,000$. The specific data of the path length between each point is shown in TABLE II.

TABLE II. PATH DIST ANCE

\begin{tabular}{|c|c|c|c|c|c|c|c|c|c|c|c|}
\hline number & 0 & 1 & 2 & 3 & 4 & 5 & 6 & 7 & 8 & 9 & 10 \\
\hline 0 & & 5 & 9 & 8 & 10 & 9 & 8 & 6 & 7 & 9 & 10 \\
\hline 1 & & & 5 & 7 & 9 & 8 & 10 & 11 & 3 & 6 & 6 \\
\hline 2 & & & & 3 & 4 & 7 & 12 & 14 & 8 & 11 & 9 \\
\hline 3 & & & & & 2 & 5 & 10 & 12 & 11 & 14 & 11 \\
\hline 4 & & & & & & 7 & 11 & 13 & 14 & 17 & 13 \\
\hline 5 & & & & & & & 5 & 7 & 12 & 15 & 12 \\
\hline 6 & & & & & & & & 8 & 11 & 14 & 11 \\
\hline 7 & & & & & & & & 11 & 14 & 15 \\
\hline 8 & & & & & & & & & & 3 & 6 \\
\hline 9 & & & & & & & & & & & 6 \\
\hline 10 & & & & & & & & & & \\
\hline
\end{tabular}




\section{3) Traffic data}

According to the periodic congestion statistics of the road, the damping factors of different paths are estimated by the driving speed of the general vehicle. The specific values are shown in TABLE III.

TABLE III. PATH DAMPING FACTOR TABLE

\begin{tabular}{|c|c|c|c|c|c|c|c|c|c|c|c|}
\hline Number & 0 & 1 & 2 & 3 & 4 & 5 & 6 & 7 & 8 & 9 & 10 \\
\hline 0 & & 2.1 & 1.8 & 1.6 & 1.8 & 1.7 & 1.2 & 1.4 & 2.2 & 2.1 & 1.8 \\
\hline 1 & & & 1.5 & 1.8 & 1.7 & 1.8 & 1.3 & 1.7 & 1.8 & 1.5 & 1.4 \\
\hline 2 & & & & 1.1 & 1.5 & 1.4 & 1.2 & 1.3 & 1.9 & 1.6 & 1.5 \\
\hline 3 & & & & & 2.6 & 1.2 & 1.3 & 1.5 & 2.2 & 1.9 & 1.6 \\
\hline 4 & & & & & & 1.7 & 1.1 & 1.2 & 2.1 & 1.8 & 1.4 \\
\hline 5 & & & & & & & 1.9 & 1.4 & 2.3 & 2.0 & 1.5 \\
\hline 6 & & & & & & & & 1.3 & 1.6 & 1.4 & 2.0 \\
\hline 7 & & & & & & & & & 1.8 & 1.7 & 1.8 \\
\hline 8 & & & & & & & & & & 1.9 & 1.7 \\
\hline 9 & & & & & & & & & & & 1.5 \\
\hline 10 & & & & & & & & & & & \\
\hline
\end{tabular}

4) Other values

Other values related to the delivery task are shown in TABLE IV, where the service time of the delivery point is taken from the average of the past delivery service time statistics of the enterprise, including the loading and unloading time. The time cost of a unit vehicle is to convert distribution efficiency into cost impact. The departure cost of a unit vehicle is the fixed cost per vehicle used by the company, including depreciation and labor costs. The unit fuel consumption cost is converted to 7 yuan $/ \mathrm{kg}$ by taking the average price of no. 0 diesel in China for nearly one year. The standard speed is the
Maximum speed limit of the vehicle on an unobstructed city road. The brand model of the distribution vehicle used by the company is: Iveco-winning, whose maximum load is 2 tons Energy consumption parameters take other scholars' research conclusions on vehicle energy consumption. Carbon trading price is taken from China's carbon trading market. Carbon emission coefficient is taken from China Energy Network and carbon quota is estimated for single distribution task.

TABLE IV. OTHER VALUES TABLES

\begin{tabular}{|c|c|}
\hline Distribution point service time $t$ & $0.25 \mathrm{~h} /$ point \\
\hline Unit vehicle time cost $\delta$ & $¥ 80 / \mathrm{h}$ \\
\hline Unit vehicle departure cost $\rho$ & $¥ 100 /$ vehicle \\
\hline Unit fuel consumption cost $\lambda$ & $¥ 7 / \mathrm{kg}$ \\
\hline Standard speed $V$ & $60 \mathrm{~km} / \mathrm{h}$ \\
\hline Maximum vehicle load $Q$ & $2000 \mathrm{~kg}$ \\
\hline Energy consumption parameter $\beta_{0}$ & 0.3 \\
\hline Energy consumption parameter $\beta_{1}$ & $8 \times 10^{-5}$ \\
\hline Energy consumption parameter $\beta_{2}$ & $2 \times 10^{2}$ \\
\hline Fuel carbon emission coefficient $e$ & $3 \mathrm{~kg}-\mathrm{CO}_{2} / \mathrm{kg}$ \\
\hline Carbon trading price $\eta$ & $Y 10 / \mathrm{kg}$ \\
\hline Carbon quota $P$ & $125 \mathrm{~kg} / \mathrm{bout}$ \\
\hline
\end{tabular}

\section{B. Problem solving}

According to the above design, the results of random operation 5 times using MATLAB under the carbon trading parameter $(P, \eta)=(125,10)$ are shown in TABLE V. The results of the five operations are the same and the average running time is $2.48 \mathrm{~s}$. The average number of iteration steps for the first time of the optimal solution is the 34th step, and the distribution task requires 4 delivery vehicles. The optimal delivery cost is 1418.65 Yuan.

TABLE V. MATLAB 5 CALCULATION RESULTS

\begin{tabular}{|l|l|l|l|l|l|l|}
\hline Calculation order & 1 & 2 & 3 & 4 & 5 & average value \\
\hline The number of iteration steps for the first optimal solution & 41 & 25 & 36 & 30 & 38 & 34 \\
\hline operation hours & 2.3 & 1.5 & 3.3 & 2.5 & 2.8 & 2.48 \\
\hline Number of delivery vehicles & 4 & 4 & 4 & 4 & 4 & 4 \\
\hline Optimal cost $(¥)$ & 1418.65 & 1418.65 & 1418.65 & 1418.65 & 1418.65 & 1418.65 \\
\hline
\end{tabular}

In this paper, the genetic algorithm is used to find the optimal solution in MATLAB. The slowest convergence rate of the fitness value calculated at the first time is shown in Fig. 2. It can be seen from the figure that the optimal solution appears in the early stage of iterative evolution, which indicates that the genetic algorithm can accurately search for the optimal solution in a short time and can be applied to the solution of the vehicle routing problem considering the carbon transaction cost.

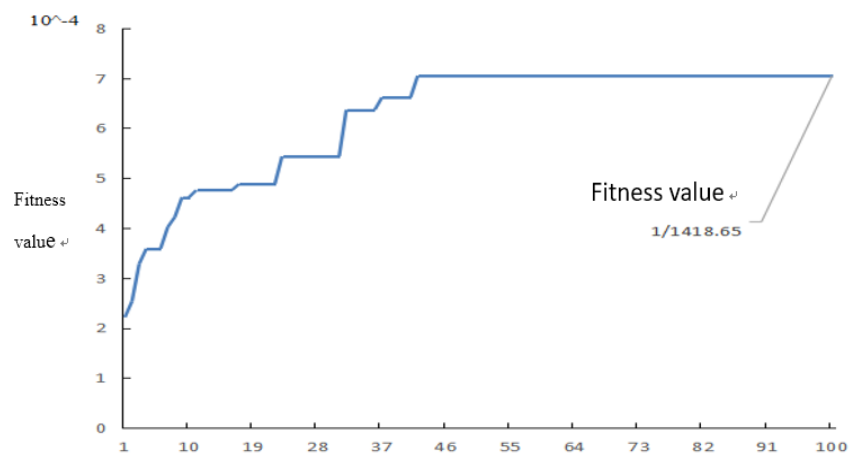

Fig. 2. Convergence graph 
The specific plan of distribution is shown in TABLE VI and TABLE VII.

TABLE VI. DISTRIBUTION PATH PLAN

\begin{tabular}{|c|c|c|c|c|c|}
\hline Distribution plan & Time costs $(¥)$ & Fuel consumption cost ( $¥)$ & Departure cost $(¥)$ & Carbon trading cost $(¥)$ & total cost $(¥)$ \\
\hline $0-3-4-2-0$ & \multirow{4}{*}{350.07} & \multirow{4}{*}{418.27} & \multirow{4}{*}{400} & \multirow{4}{*}{250.31} & \multirow{4}{*}{1418.65} \\
\hline $0-7-6-5-0$ & & & & & \\
\hline 0-8-9-0 & & & & & \\
\hline $0-1-10-0$ & & & & & \\
\hline
\end{tabular}

As can be seen from TABLE VI, $(P, \eta)=(125,10)$, the four paths completing the distribution task are 0-3-4-2-0, 0-7-6-5-0, $0-8-9-0 ., 0-1-10-0$ in the process of carbon trading parameter. Four delivery vehicles are used, the delivery task time cost is
350.07 yuan, the fuel consumption cost is 418.27 Yuan, the delivery cost is 400 Yuan, the carbon transaction cost is 250.31 yuan, and the total cost of the delivery task is 1418.65 Yuan.

TABLE VII. VEHICLE DRIVING INFORMATION TABLE

\begin{tabular}{|c|c|c|c|c|c|c|}
\hline \multicolumn{2}{|c|}{ Delivery route } & Cargo volume (kg) & Travel distance $(\mathrm{km})$ & Fuel consumption (kg) & Cumulative time (h) & carbon emission $(\mathrm{kg})$ \\
\hline \multirow{4}{*}{$0-3-4-2-0$} & $0-3$ & 1800 & 8 & 4.69 & 0.21 & 14.07 \\
\hline & $3-4$ & 1000 & 2 & 1.51 & 0.55 & 4.53 \\
\hline & $4-2$ & 600 & 6 & 2.84 & 0.95 & 8.51 \\
\hline & $2-0$ & 0 & 9 & 4.32 & 1.47 & 12.96 \\
\hline \multirow{4}{*}{$0-7-6-5-0$} & $0-7$ & 2000 & 6 & 3.41 & 0.14 & 10.24 \\
\hline & $7-6$ & 800 & 8 & 3.66 & 0.56 & 10.99 \\
\hline & $6-5$ & 300 & 5 & 2.62 & 0.97 & 7.87 \\
\hline & $5-0$ & 0 & 9 & 4.15 & 1.48 & 12.44 \\
\hline \multirow{3}{*}{$0-8-9-0$} & $0-8$ & 1900 & 7 & 5.05 & 0.26 & 15.14 \\
\hline & $8-9$ & 800 & 3 & 1.69 & 0.60 & 5.08 \\
\hline & $9-0$ & 0 & 9 & 4.91 & 1.17 & 14.72 \\
\hline \multirow{3}{*}{$0-1-10-0$} & $0-1$ & 2000 & 5 & 3.53 & 0.18 & 10.58 \\
\hline & $1-10$ & 800 & 6 & 2.84 & 0.57 & 8.51 \\
\hline & $10-0$ & 0 & 10 & 4.80 & 1.12 & 14.40 \\
\hline \multicolumn{2}{|c|}{ total } & 7700 & 93 & 50.01 & 5.23 & 150.03 \\
\hline
\end{tabular}

From TABLE VII, it can be seen that the driving status of four cars on each path is demonstrated, including the carrying weight and fuel consumption, etc. The total load of the four delivery vehicles from the distribution center is $7700 \mathrm{~kg}$, and every vehicle is not overload. The total driving distance of the vehicles returning to the distribution center after completion of the distribution task is $93 \mathrm{~km}$, the total fuel consumption is $50.01 \mathrm{~kg}$, the cumulative time is $5.23 \mathrm{~h}$, and the total carbon emission is $150.03 \mathrm{~kg}$.

The following table shows the variation curve of the total cost of the optimal path under the influence of different carbon trading parameters $P=(100,125,150,175,200)$ and $\eta=(5 \sim 15)$, as shown in Fig. 3:

From Fig. 3, we can get the following conclusions:

Effect of carbon price and carbon allowance $n$ total cost

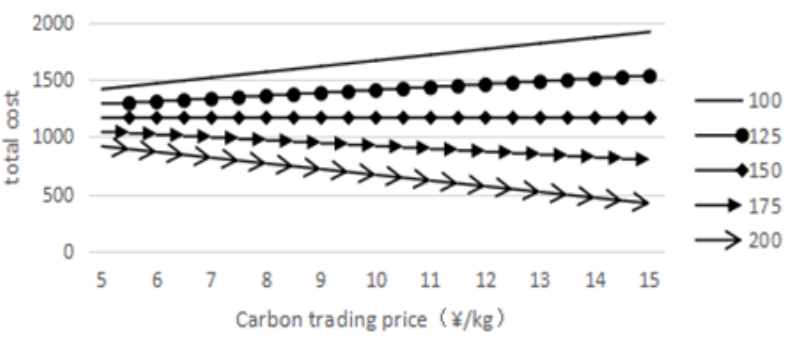

Fig. 3. Effect of carbon price and carbon allowance $n$ total cost
(1) In the case of a given carbon trading price, the total cost of the distribution task is inversely related to the growth of the carbon allowance, that is to say the higher the carbon allowance is, the lower the total cost of the distribution task is.

(2) In the case of a given carbon quotas, the total cost of the distribution task changes with the price of the carbon transaction. In the stage where the carbon quotas is less than $150.03 \mathrm{~kg}$ (the minimum carbon emission of the distribution task), the total cost of the distribution task will show gradually declined growth with the growth of carbon quotas. When the carbon quotas exceed $150.03 \mathrm{~kg}$, the total cost of distribution tasks will gradually decrease with the increase of carbon quotas.

As for vehicle routing problem under the influence of carbon trading policy, the regulation of carbon trading price and carbon quota by market and policy can also regulate the carbon emission of logistics and distribution, and enterprises have the opportunity to obtain economic benefits from emission reduction, which is beneficial to inspire enterprises to enhance their own awareness of emission reduction. Under the influence of carbon trading mechanism, the decision of logistics distribution plan should coordinate other costs and carbon transaction costs to ensure the minimum total cost of distribution, according to different carbon allowances and carbon prices 


\section{RESUlts}

This paper has established the optimization model of urban distribution route under the influence of carbon trading policy, taking comprehensive cost including carbon trading cost as its minimum goal and choosing the optimal path of enterprise urban logistics distribution. Carbon trading cost, as an important cost factor in the urban logistics distribution, can not only stimulate the subjective initiative of enterprises to reduce emissions, but also help enterprises achieve the "Independent Contribution” in the Paris Agreement to participate in energy conservation and emission reduction. At the same time, it has made a great contribution into a green and sustainable growth mode.

Due to the limited research level of the author and the limited length of the paper, there are still many directions that can be continued. For example, this paper does not apply research on other types of vehicle routing problems. And the results aren't calculated by genetic algorithm, which is not been verified by other algorithms. In the future, it can be compared with the conclusions of other algorithms, which can be used as the follow-up research content of this paper.

\section{REFERENCES}

[1] Luo Yong, Chen Zhi-ya,. Path Optimization of Logistics Distribution Based on Improved Genetic Algorithm[J]. Systems Engineering , 2012, 08: 118-122.(In Chinese)

[2] Zhu Chang-zheng, LI Yan-ling. Study on vehicle routing optimization problem based on carbon emissions minimizing[J]. Computer Engineering and Applications,2013,49(22):15-18.(In Chinese)
[3] Li Jin, Fu Pei-hua, Model and Simulation for Vehicle Routing Problem with Time Windows Based on Energy Consumption[J]. Journal of System Simulation,2013, 25 (6):1147-1154; (In Chinese)

[4] Li jin, Zhang jiang-hua, Study on the effect of carbon emission trading mechanism on logistics distribution routing decision[J].Systems Engineering-Theory \& Practice,2014,07:1779-1787. (In Chinese)

[5] Zhou Cheng, Wang Lei, Tao Jun-cheng, Research on Logistics Distribution Routing Optimization Under the Different Carbon Emission Policies[J]. Journal of Wuhan University \& Technology ( Transportation Science \& Engineering),2015,03:479-483. (In Chinese)

[6] Zhang Ru-yun, Liu Qing, CO2 Emission Minimizing for the TimeDependent VRP in Urban Area[J].Industrial Engineering and Management,2015,(04):29-34. (In Chinese)

[7] Miguel F.M.A. Vehicle routing problem for emissions minimization [J] .Transp. Res. Rec.: J. Transp. Res. Board, 2010, 2197: 1-7.

[8] Miguel F.M.A. The impacts of congestion on time-definitive urban freight distribution networks CO2 emission levels Results from a case Study in Portland, Oregon[J].Transp. Res. Part C, 2011, 19(5): 766-778.

[9] BEKTAST, LAPORTEG. The pollution - routing problem [J]. Transportation Research: Method - logical, 2011,45(8):232-1250.

[10] BRYSY, GENDREAU M. Genetic algorithms for the vehicle routing problem with time windows[R]. Oslo: Internal Report STF42 A01021, SINTEF Applied Mathematics, 2001.

[11] POWELL B J, PERAKIS A N .Fleet deployment optimization for liner shipping: An integer programming model [J].Maritime Policy and Management, 1997 (4): 34-35.

[12] Kuo Y. Using Simulated Annealing to Minimize Fuel Consumption for the Time-Dependent Vehicle Routing Problem [J]. Computers \& Operations Research (S0305-0548), 2010, 59(1): 157-165.

[13] Suzuki Y. A New Truck-Routing Approach for Reducing Fuel Consumption and Pollutants Emission [J]. Transportation Research Part D: Transport and Environment (S1361-9209, 2011, 16(1): 73-77. 